\title{
ATIVIDADES DE EDUCAÇAO EM SAÚDE PARA GESTANTES NO CÉNARIO DA ATENÇÃO PRIMÁRIA À SAÚDE: relato de experiência
}

\author{
HUBERT, Keli Astrid ${ }^{1}$ \\ WIECHORECK, Carla ${ }^{2}$ \\ TRINDADE, Letícia de Lima $^{3}$ \\ VENDRUSCOLO, Carine ${ }^{4}$ \\ ZANOTELLI, Silvana dos Santos ${ }^{5}$ \\ ZOCCHE, Denise Azambuja ${ }^{6}$
}

Resumo: Trata-se de um relato de experiência da construção e desenvolvimento de um grupo de gestantes no cenário da Atenção Primária à Saúde. O grupo de gestantes se revelou como importante estratégia de educação em saúde, essencial para garantir a assistência integral e de qualidade para ,a gestante, bebê e família Configura-se como uma excelente estratégia para acolher as gestantes e suas famílias e criar vínculos entre usuários e profissionais, contudo requer novas propostas para acolhimento das mulheres, contribuindo para fortalecer o pré-natal. Conclui-se que é importante assegura maior qualidade da assistência prestada à gestante, sendo promissor o desenvolvimento de espaços de troca de experiências, de conhecimentos e de exercício da assistência interdisciplinar.

Palavras - Chave: Atenção Primária à Saúde; Pré-Natal; Enfermagem; Educação em Saúde.

\section{INTRODUÇÃO}

\footnotetext{
${ }^{1}$ Graduanda de Enfermagem pela Universidade do Estado de Santa Catarina. Av. Fernando Machado, 827- D, Centro, Chapecó. Cep: 89802110. E-mail: keliastridh@ gmail.com

${ }^{2}$ Graduanda de Enfermagem pela Universidade do Estado de Santa Catarina. Av. Fernando Machado, 827- D, Centro, Chapecó. Cep: 89802110. E-mail: carla.wiechoreck@ gmail.com

${ }^{3}$ Doutora em Enfermagem, Docente da Universidade do Estado de Santa Catarina. Endereço: Av, Fernando Machado, 685D, ap. 1103, Chapecó/SC E-mail: letrindade@ hotmail.com Doutora em Enfermagem, Docente da UDESC. Email: carine.vendruscolo@udesc.br 4

${ }^{5}$ Mestre em Enfermagem, Docente da Universidade do Estado de Santa Catarina. Email:szanotelli@gmail.com
}

Revista Inova Saúde, Criciúma, vol.9, n. 1, jul. 2019. 
A Política Nacional de Atenção Integral à Saúde da Mulher (PNAISM) foi elaborada em 2004 pelo Ministério da Saúde (MS), a partir do diagnóstico epidemiológico da situação da saúde da mulher no Brasil, incorporando a integralidade e a promoção da saúde como princípios norteadores. A Política busca consolidar os avanços no campo dos direitos sexuais e reprodutivos, na melhoria da atenção obstétrica, no planejamento familiar, na atenção ao abortamento inseguro e no combate à violência doméstica e sexual, e reflete o compromisso com a implementação de ações de saúde que contribuam para a garantia dos direitos humanos das mulheres (BRASIL, 2004).

$\mathrm{Na}$ área da saúde da mulher, a Norma Operacional de Assistência à Saúde (NOAS) estabelece para os municípios a garantia das ações básicas mínimas de pré-natal e puerpério, planejamento familiar e prevenção do câncer de colo uterino e, para garantir o acesso às ações de maior complexidade, prevê a conformação de sistemas funcionais e resolutivos de assistência à saúde, por meio da organização dos territórios estaduais (BRASIL, 2004).

É essencial que a atenção à saúde seja elaborada numa perspectiva humanizada e com qualidade, visando ações de saúde mais eficazes para a resolução de problemas identificados, refletindo na satisfação das usuárias, no fortalecimento da capacidade das mulheres frente à identificação de suas demandas, no reconhecimento e reivindicação de seus direitos, promovendo o autocuidado. A qualidade da atenção deve estar voltada, aos aspectos psicológicos, sociais, biológicos, sexuais, ambientais e culturais, e não centrada no modelo biomédico (BRASIL, 2004).

A Atenção Primária à Saúde (APS), no Brasil chamada de Atenção Básica, foi se fortalecendo aos poucos e deve ser a porta de entrada preferencial dos usuários e mulheres no Sistema Único de Saúde (SUS), sendo o ponto de partida para a estruturação dos sistemas locais de saúde. Esta caracteriza-se por um conjunto de ações de saúde, no âmbito individual e coletivo, que abrangem a promoção e a proteção da saúde, a prevenção de agravos, o diagnóstico, o tratamento, a reabilitação e a manutenção da saúde. É o contato preferencial dos usuários com os sistemas de saúde e orienta-se pelos princípios da universalidade, da acessibilidade e da coordenação do cuidado, do vínculo e continuidade, da integralidade, da 
responsabilização, da humanização, da equidade e da participação social (BRASIL, 2006). Ainda ressalta-se que a Atenção Básica tem a Saúde da Família como estratégia prioritária para sua organização de acordo com os preceitos do SUS (BRASIL, 2006).

$\mathrm{Na}$ atenção à saúde das mulheres gestantes, a tecnologia de cuidado utilizada na APS é o cuidado pré-natal, embasado no Programa de Humanização do Pré-natal e Nascimento (PHPN, 2000) e fortalecido atualmente pela Rede Cegonha (2011). Nesse sentido, o MS (2012) considera essencial que o pré-natal se inicie precocemente na APS, iniciando no primeiro trimestre de gestação, com um número mínimo de seis consultas. No Brasil, notou-se um aumento na cobertura da assistência pré-natal desde os anos 1990, resultando em valores superiores a $90 \%$ em várias regiões do país e em mulheres com diferentes características demográficas, sociais e reprodutivas. Contudo, verificou-se menor acesso à assistência pré-natal por mulheres indígenas e negras, as com baixa escolaridade, as com maior número de gestações e pelas residentes nas regiões Norte e Nordeste, desta maneira, evidencia a persistência de desigualdades sociais no acesso das mulheres aos serviços de saúde (DOMINGUES, et al., 2015).

No cenário da Estratégia Saúde da Família (ESF) o enfermeiro desempenha ações educativas para as mulheres e suas famílias, realiza consulta de pré-natal de gestantes de baixo risco, intercaladas com as consultas médicas, solicita exames de rotina e orienta o tratamento de acordo com o protocolo do serviço, bem como encaminha gestantes identificadas como de risco para o serviço de referência, realiza atividades com grupos de gestantes, grupos de sala de espera, realiza visita domiciliar, concede o cartão da gestante, efetua a coleta de exame citopatológico (SOUZA et al., 2013), entre outros cuidados.

Contudo, as atividades se destacam, na sua maioria, como de ordem individual, sendo o trabalho/assistência em grupo uma estratégia importante para a promoção da saúde, no contexto da educação em saúde. Sendo assim, é importante que se viabilizem espaços nas Unidades Básicas de Saúde, como por exemplo, grupos de gestantes, com foco num pré-natal de qualidade. Estes podem ser considerados importantes espaços de escuta ativa e acolhimento nos serviços de saúde que assistam as mulheres gestantes. 
Por tudo isso e por vislumbrar situações cotidianas na APS que elevam a vulnerabilidade da gestante e do recém-nascido, as quais podem levar a sérias complicações obstétricas, neonatais e psicológicas para a gestante, bebê e família, percebeu-se a necessidade de construir e implantar um grupo de gestantes no cenário da APS, considerando novas metodologias de condução e horários alternativos para melhor acolhimento das usuárias do território.

O objetivo deste trabalho é relatar a construção e desenvolvimento de um grupo de gestantes no cenário da APS, discutindo e provocando a reflexão sobre suas potencialidades.

\section{A universidade e a unidades básica de saúde: movimentos de integração ensino-serviço com vistas à saúde das gestantes}

A construção do grupo de gestantes é essencial para garantir uma assistência integral e de qualidade para a gestante e família durante o pré-natal. Uma assistência de qualidade neste momento tem o objetivo de prevenir diversas patologias que ocorrem tanto na gestante, quanto no feto, favorece um preparo psicológico durante a gestação e para o parto, previne abortos, parto prematuro ou até óbito materno ou perinatal (COSTA, 2015). As mulheres gestantes precisam de informações suficientes para tomada de decisão sobre seu corpo e suas escolhas.

Pensando nisso, um grupo de gestantes foi implantado no munício de Chapecó SC, em uma Unidade Básica de Saúde com duas ESF. Cada ESF atende uma população aproximada de 4.000 pessoas e uma área possuía no momento da implantação do grupo, 25 gestantes e a outra 28, segundo dados do sistema de saúde do município (CHAPECÓ, 2016).

Segundo o IBGE a população do Município de Chapecó no ano de 2010 era de 183.530, e para 2016 foi estimada em 209.553 habitantes. Tendo um número significativo de habitantes, há no município 27 UBS, 50 ESF, o que perfaz uma cobertura de equipes de saúde da família considerada ótima, de $100 \%$ da população (IBGE, 2010). 
Nesse cenário, o trabalho em rede é fundamental no atendimento à gestante. Na UBS é realizado o acompanhamento pré-natal de todas as gestantes de baixo risco, as usuárias que têm sua gravidez considerada de alto risco são encaminhadas para fazer o acompanhamento especializado na Clínica da Mulher do próprio município, e em situações de urgências obstétricas, são encaminhadas ao pronto socorro do hospital, ou a unidade de pronto atendimento. A grande maioria das gestantes usuárias da rede pública de saúde, realizam acompanhamento na UBS, com as equipes de ESF.

A proposta fez parte da intervenção orientada pelo Estágio Supervisionado II, da $10^{\mathrm{a}}$ fase do Curso de Enfermagem da Universidade do Estado de Santa Catarina (UDESC). O Curso de Enfermagem da UDESC vem promovendo a reformulação do seu Projeto Pedagógico, com vistas a formar profissionais qualificados para atuar no SUS, sobretudo no âmbito da APS. Inclui, para tanto, atividades de integração ensino-serviço atendendo as políticas de educação do Ministério da Saúde e as Diretrizes Curriculares para os cursos da área. Tal iniciativa aproxima instituição formadora e serviços de saúde, oportunizando o processo de aprendizagem com base em vivências na realidade local, com protagonismo dos acadêmicos na rede assistencial.

A disciplina Gestão e Gerenciamento em Enfermagem, tem uma carga horária de 504 horas. Esse componente curricular busca inserir o acadêmico de enfermagem, no seu último semestre de curso, no contexto da atenção básica em saúde, a qual é considerada a principal porta de entrada das usuárias no SUS. A prática iniciou em no primeiro dia de agosto de 2016 e desenvolveu-se até dois de dezembro de 2016.

\section{A construção de um grupo de Gestantes: acolhendo e criando vínculos (Encontro I)}

A assistência pré-natal tem como meta acompanhar toda a população de gestantes, devendo ser garantido o acompanhamento integral e a continuidade do atendimento. Para tanto realiza-se busca ativa de todas gestantes para que estas iniciem o pré-natal ainda no primeiro trimestre de gestação, para que seja prevenida 
qualquer intercorrência materna e fetal, realizando-se diagnóstico e tratamento precocoes. (ARAUJO, 2013).

No primeiro momento da prática acadêmica, buscou as informações sobre as gestantes atendidas na UBS em um caderno de registro de gestantes da unidade. Com o nome das gestantes e seus dados, realizou a busca ativa no domicílio de algumas delas, para conhecer a realidade da população em questão, e perguntar se possuíam algum interesse no grupo de gestantes e quais questões referentes ao tema eram de seu interesse. Nas terças-feiras os médicos das duas ESF realizam as consultas de pré-natal (totalizando vinte atendimentos), nessa ocasião as gestantes eram abordadas e convidadas para o grupo de gestantes. Essas atividades de convite e aproximação duraram aproximadamente três meses.

Em um segundo momento, na sala de espera da UBS, realizou-se uma conversa com todas as gestantes, explicando a formação do grupo, se possuíam interesse, qual seria o melhor horário, qual número de consultas de pré-natal, idade gestacional, para que, com estas informações se definisse melhor data e horário para realização do grupo, a fim de obter-se o maior número de gestantes participantes.

A partir do levantamento dos dados, após abordar cerca de 30 gestantes, a opção foi pelo período da noite, devido a maioria das gestantes trabalhar durante 0 dia. Sendo assim, as gestantes foram abordadas em dois grupos, os quais ocorreram em duas noites, por aproximadamente três horas cada, buscando-se maior alcance das gestantes que trabalham.

As atividades contaram com o auxilio das Agentes Comunitárias de Saúde (ACS), as quais recepcionavam as gestantes e encaminhavam-nas para a sala onde acontecia o grupo. Inicialmente as gestantes foram acolhidas com um lanche, algumas dúvidas eram esclarecidas e orientava-se conforme a idade gestacional sobre o desenvolvimento fetal, alimentação durante a gestação, acompanhamento do pré-natal e também sobre parto natural ou parto cesárea. Verificava-se a caderneta para constatar se as vacinas e as consultas de pré-natal estavam em dia e, ao final, ocorria à sessão de fotos. Todas foram convidadas para o Grupo. Participaram deste grupo um total de 22 gestantes. $O$ convite também se estendia 
para aos pais ou a outro familiar de interesse da gestante, contudo presencialmente somente as mulheres participaram.

A Educação em Saúde exige do profissional de saúde, e especialmente da enfermagem, por sua proximidade com esta prática, uma análise crítica da sua atuação e reflexão sobre seu papel como educador. O educador é o profissional que utiliza as palavras e gestos como instrumento de trabalho nesta luta coletiva. $O$ enfermeiro exerce função significativa para a população, pois participa de programas e atividades de educação em saúde, visando à melhoria da saúde do indivíduo, da família e da população. Trata-se de um educador que está inserido no contexto que guia a Educação em Saúde, sendo necessário orientar a população, mostrando alternativas que melhorem as atitudes dos usuários proporcionando maior qualidade na saúde. A educação em saúde contempla todas as ações de saúde e portanto, deve estar inserida na prática diária do enfermeiro (ALKENBERG et al., 2014). Nesse sentido, o primeiro encontro fez refletir sobre o importante papel da enfermagem na educação em saúde, sobretudo no cenário da APS, pois é nesse nível de atenção que são elaboradas e implementadas as estratégias de prevenção e promoção da saúde das coletividades.

\section{Curso de gestantes (Encontro II)}

O segundo encontro aconteceu em dois momentos, no primeiro, houve a recepção e acolhimento das gestantes com uma "Dinâmica de Desafio", em que as gestantes em círculo, com uma música tocando, repassavam um envelope, no qual havia um papel com uma frase, e quando a música parasse, quem estivesse com o papel tinha a opção de abri-lo ou continuar passando. Foi feito um pequeno suspense com perguntas do tipo: Está preparada? Você vai vir aqui na frente? Quer abrir o envelope ou vamos continuar? A gestante tinha a opção de abrir o envelope ou passá-lo para a próxima participante. A música parou várias vezes e a gestante que resolveu abrir a caixa, ganhou kit de cuidados para recém-nascido. Essa dinâmica serviu para que as gestantes percebessem o quanto as pessoas têm medo de desafios, pois foi observada a pressa em se passar o envelope para a outra participante. 
As atividades continuaram com a condução da educadora física, a qual abordou o tema atividade física e postura na gestação, na sequência a enfermeira obstetra abordou sobre aleitamento materno, vantagens da amamentação, estimulando este processo com o acompanhamento de um enfermeiro, que faça orientações sobre como deve ser o posicionamento para ter uma boa pega e evitar assim, traumas mamilares. E finalizando o grupo, a nutricionista realizou orientações referente ao tema alimentação saudável na gestação.

$\mathrm{Na}$ segunda noite, os profissionais convidados foram, enfermeira obstetra que falou sobre parto e cesárea, deixando-as confortáveis para decidirem a via de parto que se sentirão mais seguras, vantagens e desvantagem do parto e cesariana. Essa abordagem buscou o maior empoderamento das gestantes a partir do compartilhamento de informações sobre o parto. Posteriormente a médica da família abordou alguns sintomas da gravidez que são citados como normais e outras como um indicativo de que algo não está acontecendo como deveria, relatou como ocorre o funcionamento da unidade na solicitação de exames, pré-natal e cuidados com o $\mathrm{RN}$ e a enfermeira da família alertou as mulheres para que mantenham em dia o seu próprio calendário de vacinas, além da importância das vacinas que o bebê deve fazer após o nascimento.

A gravidez é uma experiência única para a mulher. É um momento de intensas transformações, com mudanças físicas e emocionais, que exige inúmeros cuidados de promoção em saúde. O período pré-natal é considerado o primeiro passo para o parto e nascimento humanizados, o qual requer dos profissionais envolvidos, o estabelecimento de um vínculo junto as gestantes e suas famílias. É preciso entender a humanização como prática pautada em princípios como integralidade e equidade das ações, evidenciando os usuários como sujeitos de direitos e participantes ativos do seu processo saúde/doença (DE SOUZA; ROECKER; MARCON, 2011).

Compreende-se que o pré-natal adequado incide na diminuição dos casos de morbimortalidade materno-infantil, isso também é evidenciado, em melhor crescimento intrauterino, maior peso ao nascer, menor ocorrência de prematuridade, de mortalidade neonatal e, por parte da mãe, em menor índice de intercorrências no período gestacional e de complicações no decorrer do parto. Aborda-se um conjunto 
de medidas essenciais à redução da morbimortalidade e da promoção do bem-estar materno-infantil (GOMES; CÉSAR, 2013).

Nos últimos anos, percebe-se a melhoria nos indicadores de assistência à saúde materno-infantil no Brasil. A taxa de mortalidade infantil no país foi reduzida a menos da metade ao longo de 13 anos, passando de 33,5 óbitos para cada 1.000 nascidos vivos em 1998 para 16,1/1.000 nascidos vivos em 2011. Segundo a literatura, isso decorre da ampliação dos serviços básicos de saúde, da melhor assistência à gestação, ao parto e ao puerpério, especialmente com a implantação da Estratégia Saúde da Família (ESF) (DOMINGUES, et al., 2015). No entanto, o componente neonatal da mortalidade infantil não teve redução na mesma proporção, o que reafirma a necessidade de atenção qualificada no pré-natal e nascimento (VICTORA, et al., 2011)

Os encontros contaram com sorteios de brindes, entrega das fotos para cada gestante e lanches, para melhor acolher as gestantes.

O grupo possui como objetivos a redução de riscos durante a gestação, melhorar a qualidade e a eficiência da assistência pré-natal e a frequência da gestante nas consultas pré-natais. Segundo orientações do MS a assistência ao prénatal é o primeiro passo para o parto e o nascimento humanizado (SOUZA et al., 2013).

Ações educativas devem ser realizadas no decorrer de todas as etapas do período gravídico puerperal, mas é no pré-natal que a mulher deverá ser melhor orientada para que possa viver o parto de forma positiva, ter menos riscos de complicações no puerpério e mais sucesso na amamentação, sendo relevante o grupo/curso de gestantes na Unidades Básicas de Saúde, necessitando ser de fácil acesso para que haja a construção do vínculo pela possibilidade da participação das mulheres em todos ou em grande parte dos encontros. A gravidez é o melhor momento para que as atividades preventivas sejam assumidas, pois os pais estão motivados para obter informações e realizar cuidados com a saúde já que este refletirá na saúde do filho (DE SOUZA; ROECKER; MARCON, 2011). Nesse sentido, reconhece-se como limites da proposta a maior participação dos pais e demais membros da família, o que requer também ações singulares e ao encontro das necessidades desse outros importante sujeitos do núcleo familiar. 


\section{CONSIDERAÇÕES FINAIS}

O pré-natal e o nascimento são considerados como momentos únicos para cada mulher, com isso, os profissionais de saúde, e sobretudo a enfermagem, devem assumir a postura de educadores compartilhando saberes. Aos acadêmicos, oportunizou-se a vivência profissional através da responsabilidade de planejar, coordenar e organizar as atividades grupais, além de desenvolverem a habilidades de comunicação e refletirem possibilidades de prevenir problemas gestacionais e promover a saúde das gestantes, mediante a realidade local.

Os momentos vividos possibilitaram conhecer, compreender e identificar a transformação da realidade. Esse processo ocorreu mediante uma construção coletiva através do diálogo, para identificar, aprender, criar, compartilhar e refletir sobre as potencialidades e limitações referentes à saúde integral individual-coletiva das gestantes. Atividades como estas podem contribuir para 0 maior empoderamento das mulheres e de suas família para escolha da via de parto, cuidados com o bebê e outras questões emergentes no nascimento do bebê.

Ainda, o grupo de gestantes proporcionou momentos de ampla aprendizagem para as participantes e para as organizadoras, reafirmando a ideia de que as ações de promoção da saúde são fundamentais para a qualificação da APS. Importante lembrar que atuar em promoção de saúde significa criar possibilidades de intervenção, enfocando a saúde como qualidade de vida e envolvendo o usuário. Nessa direção, a educação em saúde opera como uma importante estratégia para promover a saúde.

Reconhece-se que a vivência alcança um único território e potencialmente, poderiam incluir mais membros das famílias das gestantes, o que se destaca como uma limitação das atividades relatadas, contudo o significado destas pode contribuir e inspirar novas propostas de melhor acolhimento, extrapolando as ações programáticas e prescritivas comuns na APS para essa etapa singular na vida das mulheres e suas famílias.

\section{REFERÊNCIAS}


Alkenberg, et al. Educação para a saúde e educação no sistema de saúde: conceitos e implicações para a saúde pública. Ciência \& Saúde Coletiva.2014; 19(3):847-82.

Araujo,SM, et al. A importância do pré-natal e a assistência de enfermagem. Veredas Favip-Revista Eletrônica de Ciências. 2013; 3(2): sp

Ministério da Saúde (BR). Secretaria de Atenção à Saúde. Departamento de Ações Programáticas Estratégicas. Política nacional de atenção integral à saúde da mulher: princípios e diretrizes. Brasília: Ministério da Saúde; 2004.

Ministério da Saúde (BR). Secretaria de Atenção à Saúde. Departamento de Atenção Básica. Política nacional de atenção básica. Brasília: Ministério da Saúde; 2006.

Ministério da Saúde (BR). Manual de estrutura física das unidades básicas de saúde: saúde da família. 2. ed. Brasil: Ministério da Saúde; 2008. (Série A. Normas e Manuais Técnicos).

Costa CSC, et al. Características do atendimento pré-natal na Rede Básica de Saúde. Rev eletr enf. 2013;15(2):516-22.

Souza VB, Roeckerii S, Marconi SS. Ações educativas durante a assistência prénatal: percepção de gestantes atendidas na rede básica de Maringá-PR. Revista Eletrônica de Enfermagem. 2011; 13(2):199-210.

Domingues RMSM, et al. Adequação da assistência pré-natal segundo as características maternas no Brasil. Rev Panam Salud Publica. 2015; 37(3):140-7.

Victora CG, Aquino EM, Leal MC, Monteiro CA, Barros FC, Szwarcwald CL. Maternal and child health in Brazil: progress and challenges. Lancet. 2011;377(9780):1863-76. 
Artigo Original

Atenção à saúde

Gomes RMT, César JA. Perfil epidemiológico de gestantes e qualidade do pré-natal em unidade básica de saúde em Porto Alegre, Rio Grande do Sul, Brasil. Rev Bras Med Fam Comunidade. 2013; 8(27):80-9.

Souza NA, et al. Perfil epidemiológico das gestantes atendidas na consulta de prénatal de uma unidade básica de saúde em São Luís-MA. Rev. Ciênc. Saúde. 2013; 5(1):28-38. 